\title{
Systemic cytokine response in patients with community-acquired pneumonia
}

\author{
H. Endeman*,\#, , S.C.A. Meijvis*, ", G.T. Rijkers", H. van Velzen-Blad", \\ C.H.M. van Moorsel ${ }^{+}$, J.C. Grutters ${ }^{+}$and D.H. Biesma ${ }^{\S}$
}

ABSTRACT: The role of individual cytokines and polymorphisms in pneumonia has been described, but the relationship between different cytokines and polymorphisms in relation to causative microorganisms, antibiotics, corticosteroids and clinical course has not. This study questions the relationship between cytokines, polymorphisms and clinical characteristics of pneumonia.

Patients diagnosed with pneumonia were included in the study. Serum cytokine levels were measured during hospital stay, genotyping was performed, causative microorganisms were identified and patients were monitored throughout the hospital stay.

In 201 patients with pneumonia interleukin (IL)-1 receptor antagonist (IL-1RA), IL-6, IL-8 and IL-10 acted as acute phase proteins. After admission, the levels of these cytokines decreased rapidly. Single nucleotide polymorphisms did not influence cytokine production and were not associated with clinical outcome. Cytokine serum levels were significantly higher in patients with pneumococcal pneumonia. The decrease in levels of cytokines was independently influenced by the start of corticosteroid therapy.

IL-1RA, IL-6, IL-8 and IL-10 are acute phase proteins, independent of genotype. Their levels are influenced by the nature of the causative microorganism and the start of corticosteroids therapy.

KEYWORDS: Chemokines, corticoids, cytokines, pneumonia, polymorphism

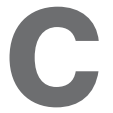
ommunity-acquired pneumonia (CAP) is the leading cause of community-acquired infection requiring hospitalisation and has an overall mortality of $8.7 \%$ [1]. The human immune response to CAP varies between the different causative microorganisms and severities of disease. An important early component of the host response is the release of cytokines produced by inflammatory cells [2, 3]. In experimental settings, the appearance of cytokines depends on the time after induction of endotoxaemia. The role of each specific cytokine in the inflammatory response to CAP is still a subject of ongoing research. In general, tumour necrosis factor (TNF)- $\alpha$ and interleukin (IL)-6 are considered essential pro-inflammatory proteins and IL-10 is considered the most important cytokine with anti-inflammatory properties [2, 4-7]. These three cytokines are reported to play an important role in the susceptibility to experimental pneumonia caused by different pathogens [8-10]. Chemokines are locally produced cytokines and their main function is to attract and activate macrophages and monocytes, thereby involving them in the primary clearance of various bacteria $[11,12]$.

In sepsis, systemic cytokine levels are associated with the severity of disease [13, 14]. In bacterial pneumonia the cytokine response is mostly confined to the affected lung [15-18], but systemic levels of cytokines are also raised [15-17, 19, 20]. The severity of pneumonia is both reflected and predicted by higher levels of cytokines in blood [16, 19-22]. Polymorphisms within the genes coding for cytokine expression are also associated with severity of disease [14, 23-29].

The role of individual cytokines and cytokine polymorphisms is well known. Herein, we question the relationship between cytokines, polymorphisms and clinical characteristics of CAP. Therefore, we describe the systemic cytokine response of different cytokines and cytokine polymorphisms in CAP in relation to causative microorganisms, the use of antibiotics and corticosteroids and clinical course. Furthermore, the role of cytokine polymorphisms on cytokine levels in CAP is reported.

\section{AFFILIATIONS}

Depts of *Internal Medicine,

"Medical Microbiology and

Immunology,

+Pulmonology, St. Antonius Hospital, Nieuwegein,

\#Dept of Intensive Care Medicine.

Diakonessenhuis, and

${ }^{\S}$ Dept of Internal Medicine, University Medical Center Utrecht, Utrecht,

The Netherlands.

${ }^{f}$ Both authors contributed equally to the study

\section{CORRESPONDENCE}

H. Endeman

Dept of Intensive Care Medicine

Diakonessenhuis

P0 Box 80250

3508TG Utrecht

the Netherlands

E-mail: henrik.endeman@planet.nl

Received:

May 112010

Accepted after revision:

Sept 082010

First published online:

Sept 302010 


\section{METHODS}

\section{Patients and controls}

From October 2004 until August 2006, patients with confirmed pneumonia admitted to the emergency department (ED) of the St. Antonius Hospital (Nieuwegein, the Netherlands) were included in this study. Pneumonia was defined as a new infiltrate on chest radiograph in combination with at least two of the following criteria: cough, sputum production, temperature $>38^{\circ} \mathrm{C}$ or $<35^{\circ} \mathrm{C}$, auscultatory findings consistent with pneumonia, C-reactive protein $(\mathrm{CRP})>15 \mathrm{mg} \cdot \mathrm{L}^{-1}$, or leukocytosis or leukopenia (white blood cell count $>10$ cells $\times 10^{9} \cdot \mathrm{L}^{-1}$ or $<4$ cells $\times 10^{9} \cdot \mathrm{L}^{-1}$, respectively, or $>10 \%$ rods in leukocyte differentiation) [30]. Patients with defined immunodeficiencies (a known congenital or acquired immunodeficiency, chemotherapy within the last 6 weeks, corticosteroids use in

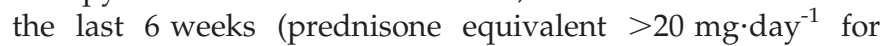
$>3$ days), immunosuppressive medication in the last 6 weeks), haematological malignancies or recent hospitalisation ( $<30$ days) were excluded. At inclusion, clinical and laboratory parameters were recorded and the Pneumonia Severity Index (PSI) was calculated [31]. Blood was collected and serum samples stored to determine cytokine profiles by multiplex immunoassays. Mortality, intensive care unit (ICU) admission, length of hospital stay and the causative microorganism were assessed. Written informed consent was obtained from all patients. The study was approved by the institutional medical ethical committee (Verenigde Commissie Mensgebonden Onderzoek, St. Antonius Ziekenhuis, Nieuwegein, the Netherlands).

\section{Pathogen identification}

The diagnostic tools used to identify the causative microorganism of CAP have been described previously [32]. In short, at least two sets of separate blood and sputum samples from each patient were cultured. Urine antigen tests were performed for the detection of Legionella pneumophila serogroup 1 and Streptococcus pneumoniae. In-house developed PCR were performed on the sputum to detect L. pneumophila, Mycoplasma pneumoniae and Chlamydophila pneumoniae/psittaci. Serological testing was performed for the presence of antibodies to M. pneumoniae, Coxiella burnetii or respiratory viruses (influenza and parainfluenza viruses, adenovirus and respiratory syncytial virus). Pharyngeal samples were taken for viral culture (influenza viruses).

\section{Determination of systemic cytokine level}

Systemic circulating concentrations of IL-1 receptor antagonist (IL-1RA), IL-5, IL-6, IL-8, IL-10, IL-12, interferon (IFN)- $\gamma$, macrophage inflammatory protein (MIP) and monocyte chemoattractant protein $(\mathrm{MCP})$ were measured on day of presentation and subsequent samples were drawn at 08:00 h on days 2, 3, 5 and 10 and at a control visit at least 30 days after admission (convalescent phase). For technical details of analysis of cytokine levels see the online supplementary material. To analyse whether these cytokines and chemokines act as acute phase proteins, we analysed the difference in cytokine levels between the acute and convalescent phases. An acute phase response was defined as a decrease or increase in the cytokine or chemokine level by at least $25 \%$ in the acute phase compared with the convalescent phase [33].

\section{DNA isolation and genotyping}

A $200-\mu \mathrm{L}$ whole blood sample was used to extract DNA with the MagNA Pure LC DNA isolation kit I (Roche Diagnostics, Mannhein, Germany) for genotyping. Genotyping was performed on a 7500 Fast Real-Time PCR System (Applied Biosystems, Foster City, CA, USA) using the TaqMan technique with customised primers and probes designed by Applied Biosystems using the manufacturer's instructions. Technical details of genotyping, including primer sequences are given in the online supplementary material. Individual haplotypes were inferred using PHASEv2 software and analysed to determine when frequencies exceeded a 5\% threshold [34]. Distribution of genotypes was compared with the distribution of genotypes in 313 healthy unrelated Dutch volunteers with the same geographical background as the patients.

\section{Statistical analyses}

All statistical analyses were performed using statistics software SPSS version 13.0 for Windows (SPSS, Inc., Chicago, IL, USA) and a two-tailed $\mathrm{p}$-value of $<0.05$ was considered significant.

Differences in continuous variables were tested by the t-test (tested within patients, paired; tested between patients, unpaired) or the Mann-Whitney U-test, as appropriate. Genotype frequencies of CAP patients were tested for conformity to Hardy-Weinberg equilibrium using the Chi-squared test between the observed and expected numbers. Correlations were tested using the Spearman's test. We conducted a linear regression analysis including age, sex, PSI score, chronic obstructive pulmonary disease (COPD), causative microorganism (pneumococcal versus nonpneumococcal), antibiotic therapy prior to hospitalisation and the use of corticosteroids and IL-6 concentration on day 1 to predict IL- 6 concentration on day 3 (dependent variable). For this linear regression the cytokines were transformed into a natural log scale because the cytokines were not normally distributed. In the linear regression analysis we chose to look at the decrease in IL-6 from day 1 to day 3 because all patients using corticosteroids were on corticosteroids for at least $24 \mathrm{~h}$ on day 3 and because cytokine concentrations decreased the most in the first few days. To correct for the height of IL- 6 on day 1 this is an independent variable in the linear regression. A similar multivariate analysis was performed to search for an effect of polymorphisms on cytokine levels, including age, comorbidities (COPD and heart failure) and PSI score.

\section{RESULTS}

In 201 patients, a new infiltrate on chest radiograph was confirmed by an experienced radiologist. We obtained blood for the assessment of cytokine concentrations from 171 of the 201 enrolled patients. DNA was isolated from 200 of the 201 patients with CAP. The baseline characteristics of these patients are shown in table 1 . In $64 \%$ of the patients the causative microorganism for the CAP could be identified.

\section{Cytokine and chemokine serum levels at admission and during hospital stay}

The levels of the nine cytokines and chemokines (and CRP) were determined at admission and on consecutive days. Figure 1 shows that the levels of IL-1RA, IL-6, IL-8, IL-10 and MCP were highest at admission. An acute phase response was observed for IL-6 $(+1695 \%$ as compared with day 30 


\begin{tabular}{lc} 
TABLE 1 & $\begin{array}{c}\text { General characteristics of } 201 \text { hospitalised } \\
\text { patients with community-acquired pneumonia }\end{array}$ \\
Age yrs & $63.7 \pm 17$ \\
Males & $124(62)$ \\
Comorbidity & \\
Diabetes mellitus & $35(17)$ \\
CVA & $17(9)$ \\
COPD & $63(31)$ \\
Hypertension & $38(19)$ \\
Nursing home residents & $3(1)$ \\
Pneumonia Severity Index & \\
Low I & $30(15)$ \\
Low II & $34(17)$ \\
Low III & $53(26)$ \\
Moderate IV & $56(28)$ \\
High V & $28(14)$ \\
ICU admission & $21(10)$ \\
Mortality & $10(5)$ \\
Microbiological species & \\
Streptococcus pneumoniae & $60(30)$ \\
Haemophilus influenzae & $14(7)$ \\
Legionella pneumophila & $9(4)$ \\
Mycoplasma pneumoniae & $9(4)$ \\
Other & $36(18)$ \\
Unknown & $73(36)$ \\
\hline &
\end{tabular}

Data are presented as median \pm SD or $n(\%)$. CVA: cerebrovascular accident (stroke); COPD: chronic obstructive pulmonary disease; ICU: intensive care unit.

levels), CRP (+1088\%), IL-1RA (+450\%), IL-10 (+332\%) and IL-8 $(+96 \%)$ (fig. 2). For MCP (+22\%) there was a trend towards an acute phase response. In contrast to acute phase cytokines, the levels of CRP remained at a high level at day $2\left(199 \mathrm{mg} \cdot \mathrm{L}^{-1}\right.$ and $201 \mathrm{mg} \cdot \mathrm{L}^{-1}$ at days 1 and 2, respectively) and decreased thereafter. Levels of the other measured cytokines and chemokines did not show an acute phase response after admission and during the hospital stay: IL-12 (+6\%), IFN- $\gamma(+6 \%)$, IL-5 (0\%) and MIP $(-4 \%)$. We analysed if there were differences in cytokine response between survivors and a combined group of patients (nonsurvivors and patients who needed admission to the ICU); however, due to lack of power (only 10 patients deceased and 21 patients were admitted to the ICU) we were unable to show statistically significant differences. We did not find a relationship between cytokine levels and/or kinetics and length of hospital stay.

\section{Polymorphisms}

An analysis of 200 patients with CAP for single nucleotide polymorphisms (SNPs) in the IL-6, IL-8, IL-10, IL-18, TNF- $\alpha$ and RANTES genes was performed. The group did not deviate significantly from the Hardy-Weinberg equilibrium ( $p>0.05)$. We did not find differences in distribution of the tested SNPs between patients and a control group of Dutch healthy unrelated volunteers (table 2). We did not find differences within patients for clinical course defined as "need of ICU admission", "in-hospital mortality" and "length of hospital stay" between the different tested genotypes.
We found no relationship between cytokine levels and polymorphisms, even in multivariate analysis correcting for age, comorbidities and PSI score. The data do not indicate significantly higher cytokine production associated with a specific SNP of the IL-6, IL-8 or IL-10 gene. The three SNPs in the IL-10 gene produced four haplotypes $(-1082,-592,+3367$ : ACG, AAG, GCG and GCA). Each of those IL-10 haplotypes showed similar magnitudes and kinetics of IL-10 production.

\section{Causative microorganisms}

We compared the serum cytokine profiles of patients with pneumococcal pneumonia with patients with another identified microorganism. After adjustments for age, PSI and the use of corticosteroids, IL-6 levels on admission were significantly higher in patients with pneumococcal pneumonia compared with nonpneumococcal pneumonia $(\mathrm{p}<0.01)$ (fig. 3). Similar results were found for the anti-inflammatory cytokine IL-1RA on admission $(\mathrm{p}<0.01)$. During the subsequent days, IL-6 and IL-1RA concentrations fell rapidly to the same (elevated) levels of patients with nonpneumococcal pneumonia.

The other cytokine levels did not differentiate between patients with pneumococcal and nonpneumococcal pneumonia at admission or at the following sample days. Results were similar when we compared pneumococcal pneumonia versus atypical pneumonia (L. pneumophila, M. pneumoniae and C. pneumoniae/psittaci).

\section{Antibiotics}

We combined the causative microorganisms found in 128 patients with the antibiotics used and scored the antibiotic therapy as being either appropriate or inappropriate (see the online supplementary material for definitions). After admission, $86 \%$ of the patients were treated with an appropriate antibiotic. At days 2, 3 and 5 this number increased to $86 \%$, $89 \%$ and $92 \%$, respectively. We did not find any differences in the cytokine levels between patients treated with appropriate or inappropriate antibiotics. The use of antibiotics prior to admission had no effect on the levels of cytokines, except for the serum levels of IL-6 at admission $(p=0.04)$, but this effect was lost after multivariate analysis combining antibiotic therapy prior to hospitalisation, PSI score and causative microorganism.

\section{Corticosteroids}

Of the 201 enrolled patients, 35 patients were using a corticosteroid upon admission (mean prednisone equivalent

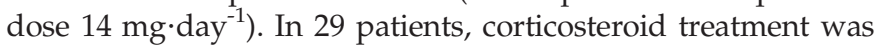
initiated on the day of admission to the hospital. In total, corticosteroid therapy (mean prednisone equivalent dose $43 \mathrm{mg} \cdot \mathrm{day}^{-1}$ ) was prescribed for 62 patients after admission (in two patients corticosteroid therapy was stopped at admission). Patients who were treated with corticosteroids were significantly older (median age 72.5 yrs versus 62 yrs, $p<0.01$ ) and more frequently known to have a history of COPD (74\% versus $11 \%$, $\mathrm{p}<0.01)$. The PSI also differed between the two groups: the median PSI score was significantly higher in patients treated with corticosteroids compared with patients who were not treated with corticosteroids (102 versus $77, \mathrm{p}<0.01$ ).

Median serum IL-6 levels were similar on the day of admission but decreased more rapidly by day 3 (at least $24 \mathrm{~h}$ on 

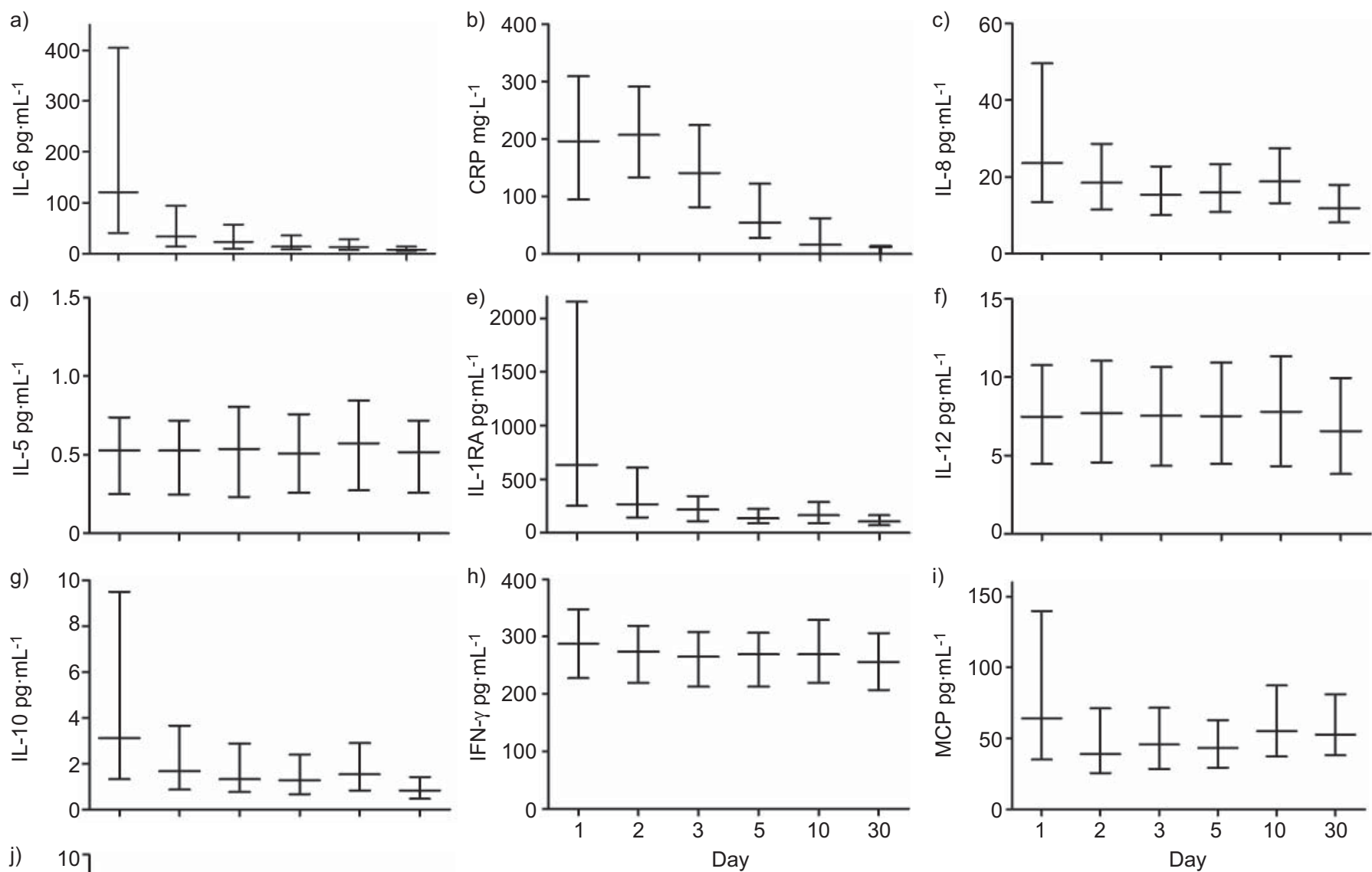

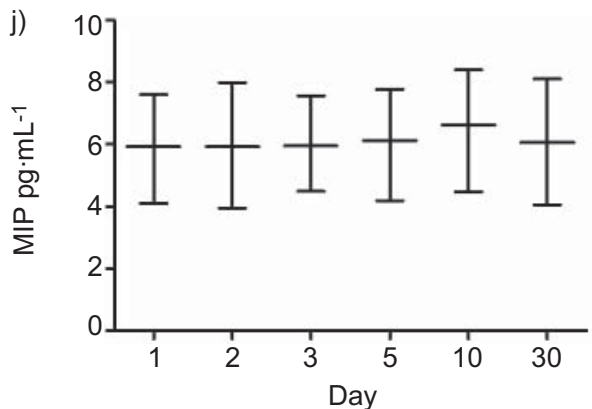

corticosteroids) for the corticosteroid treated group (fig. 3a). On days 2, 3, 5 and 10 the median IL-6 levels were significantly lower in patients treated with corticosteroids. On the day of the control visit, median IL-6 levels had returned to baseline levels and did not differ between both groups. After adjusting for age, sex, PSI score, COPD, IL-6 level on day 1 and pneumococcal pneumonia, corticosteroid therapy independently influenced IL-6 levels on day $3(p<0.01, \beta:-1.23)$. Where $\exp (-1.23)=0.29$, mean IL-6 levels were $70 \%$ less for patients using corticosteroids. Similar results were found for the IL-1RA and MCP. IL-10 levels in patients treated with corticosteroids were significantly higher at admission. Therefore, in the multivariate analysis we found no significant effect of corticosteroid therapy on IL-10 levels on day 3 . There was no effect of corticosteroid treatment on ICU admission or mortality.

Patients not receiving corticosteroids on a chronic basis could be divided into two groups who either started with corticosteroids $\leqslant 5$ days before admission or who started corticosteroids after admission (fig. 3b). Age, sex, PSI and history of COPD did not
FIGURE 1. Median plasma cytokine concentrations with the interquartile range on subsequent days. a) Interleukin (IL)-6, b) C-reactive protein (CRP), c) IL-8, d) IL-5, e) IL-1 receptor antagonist (IL-1RA), f) IL-12, g) IL-10, h) interferon (IFN)- $\gamma$, i) monocyte chemoattractant protein (MCP) and j) macrophage inflammatory protein (MIP).

differ between these groups. There were no differences in the IL-6 levels at admission or kinetics of the response between the two groups. There were no differences in the IL-6 levels at admission or kinetics of the response between patients who started corticosteroids $\leqslant 5$ days before admission and patients who started corticosteroids on admission. Both groups had a significant lower IL-6 level on day 3 compared with patients without the use of corticosteroids.

\section{DISCUSSION}

We report a comprehensive analysis of the systemic cytokine response during the clinical course of CAP in relation to cytokine polymorphisms, causative microorganisms, antibiotics, use of corticosteroids and clinical course. The major findings of this study are that in patients with CAP, serum IL-6, IL-8, IL-1RA and IL-10 act as acute phase proteins. After admission, the levels of these cytokines decreases rapidly, but SNPs in the IL-6, IL-8 and IL-10 genes (including haplotypes) do not influence cytokine production, and no association was found between cytokine polymorphisms and clinical outcome. 


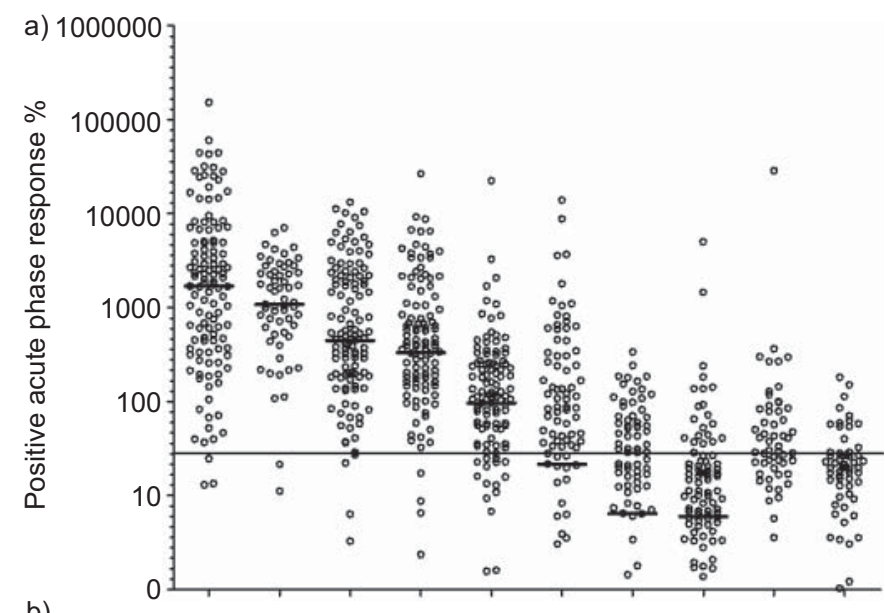

b)

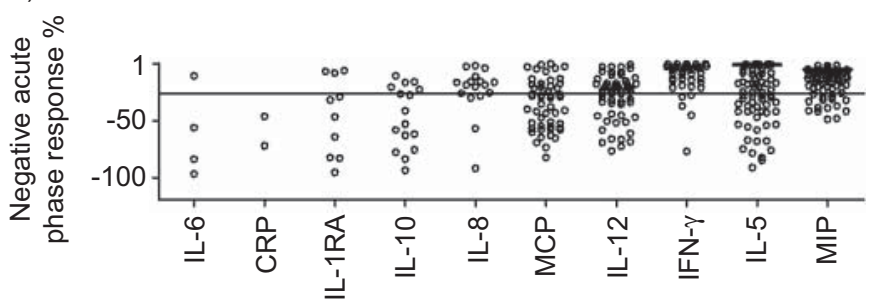

FIGURE 2. Dynamics of the measured cytokines in community-acquired pneumonia patients. The relative change (\%) of the cytokines in the acute phase of disease compared with the convalescent phase is plotted. The cut-off for positive $(+25 \%)$ and negative $(-25 \%)$ acute phase responses are plotted $(-)$. Data points of individual patients are shown as $(\bigcirc)$; horizontal bars indicate median values. a) Positive acute phase responses on a logarithmic scale and b) negative acute phase responses on a linear scale. IL: interleukin; CRP: C-reactive protein; IL-1RA: IL-1 receptor antagonist; MCP: monocyte chemoattractant protein; IFN: interferon; MIP: macrophage inflammatory protein.

Distribution of cytokine polymorphisms did not differ between patients (with different causative microorganisms) and healthy volunteers. IL-6 and IL-1RA levels are significantly higher at admission in patients with pneumococcal pneumonia. Furthermore, the decrease in levels of IL-6, IL-1RA and MCP on consecutive days was independently influenced by the start of corticosteroid therapy. IL-6 and IL-1RA levels are significantly higher at admission in patients with pneumococcal pneumonia.

Next to CRP, IL-6, IL-1RA, IL-10 and IL-8 were identified as acute phase proteins. No acute phase response was observed for IL-5, IL-12, IFN- $\gamma$, MCP and MIP. Our results are comparable with former findings in human and experimental pneumococcal pneumonia, in which the inflammatory response is characterised by an extended pro-inflammatory response resulting in high levels of IL-6 and IL-1RA [2, 10, 13, 21, 35-37]. In this study, cytokines and chemokines were only measured in serum. It might be possible that a number of cytokines, and especially chemokines, are up-regulated in the lung itself but not in the systemic circulation [15]. Our results are limited to the systemic inflammatory response as measured by serum cytokines.

Admission to the hospital marks initiation of treatment by administration of intravenous antibiotics in nearly all patients. In $86 \%$ of the patients with a known causative microorganism,

\begin{tabular}{|c|c|c|c|}
\hline \multirow{2}{*}{$\begin{array}{l}\text { TABLE } 2 \\
\text { SNP (RS- } \\
\text { number) }\end{array}$} & \multicolumn{3}{|c|}{$\begin{array}{l}\text { Distribution of polymorphisms in patients with } \\
\text { community-acquired pneumonia (CAP) and } \\
\text { healthy unrelated volunteers }\end{array}$} \\
\hline & Genotype & CAP $^{\#}$ & Controls" \\
\hline \multirow{3}{*}{$\begin{array}{l}\text { IL-6 -174 G/C } \\
\quad(\text { RS1800795) }\end{array}$} & $\mathrm{GG}$ & $83(42)$ & $113(36)$ \\
\hline & GC & $92(46)$ & $150(48)$ \\
\hline & CC & 25 (13) & $48(15)$ \\
\hline \multirow{3}{*}{$\begin{array}{l}\text { IL-6 -572 G/C } \\
\quad \text { (RS1800796) }^{+}\end{array}$} & GG & $184(92)$ & 283 (91) \\
\hline & GC & 15 (8) & $28(9)$ \\
\hline & $\mathrm{CC}$ & 1 (1) & 0 \\
\hline \multirow{3}{*}{$\begin{array}{c}\text { IL-8 -251 T/A } \\
(R S 4073)^{\S}\end{array}$} & $\mathrm{TT}$ & $46(23)$ & $62(20)$ \\
\hline & TA & $94(47)$ & $153(49)$ \\
\hline & AA & 59 (29) & 98 (31) \\
\hline \multirow{3}{*}{$\begin{array}{r}\text { IL-10 -592 C/A } \\
\text { (RS1800872) }\end{array}$} & $\mathrm{CC}$ & $115(57)$ & $173(55)$ \\
\hline & CA & 70 (35) & $126(40)$ \\
\hline & $A A$ & $15(8)$ & $14(5)$ \\
\hline \multirow{3}{*}{$\begin{array}{c}\text { IL-10 -1082 G/A } \\
\text { (RS1800896) }\end{array}$} & $G G$ & $54(27)$ & $74(24)$ \\
\hline & GA & $90(45)$ & $170(54)$ \\
\hline & $A A$ & $56(28)$ & 69 (22) \\
\hline \multirow{3}{*}{$\begin{array}{c}\text { IL-10 +3367 G/A } \\
\text { (RS3024495) }\end{array}$} & $G G$ & $134(67)$ & 225 (72) \\
\hline & GA & $63(31)$ & 77 (25) \\
\hline & AA & $3(2)$ & $11(3)$ \\
\hline \multirow{3}{*}{$\begin{array}{c}\text { IL-18 -137 G/C } \\
\text { (RS187238) }\end{array}$} & $G G$ & $109(55)$ & 168 (54) \\
\hline & GC & $79(40)$ & 121 (39) \\
\hline & CC & $12(6)$ & $24(8)$ \\
\hline \multirow{3}{*}{$\begin{array}{c}\text { TNF- } \alpha-238 \text { G/A } \\
\text { (RS361525) }\end{array}$} & GG & 187 (93) & 286 (91) \\
\hline & GA & $12(6)$ & 29 (8) \\
\hline & $A A$ & $1(1)$ & $1(0)$ \\
\hline \multirow{3}{*}{$\begin{array}{c}\text { RANTES } 1.1 \mathrm{G} / \mathrm{A} \\
\text { (RS2280789) }\end{array}$} & $G G$ & 3 (2) & $10(3)$ \\
\hline & GA & $51(25)$ & $76(24)$ \\
\hline & AA & $146(73)$ & 227 (73) \\
\hline
\end{tabular}

Data are presented as $n$ (\%). SNP: single nucleotide polymorphism; IL: interleukin; TNF: tumour necrosis factor; RANTES: regulated on activation, normal T-cell expressed and secreted. ${ }^{\#}: \mathrm{n}=200 ;{ }^{\bullet}: \mathrm{n}=313{ }^{+}{ }^{+}$: two controls missing, ${ }^{\text {s: }}$ one patient missing.

an appropriate antibiotic therapy suitable for eliminating the causative microorganism was started in our study. Effective tissue concentrations were assured by administering the antibiotics intravenously, so this is the most probable explanation for the decrease in cytokines. The sharp decrease in IL-6, IL-8, IL-1RA and IL-10 within the first 2 days of admission is in contrast with the more blunt kinetics of the $\mathrm{CRP}$ response. In daily practice, $\mathrm{CRP}$ is usually regarded as the most important acute phase protein in CAP. It should be noted that the dynamic changes in IL-6 occur earlier than CRP. 

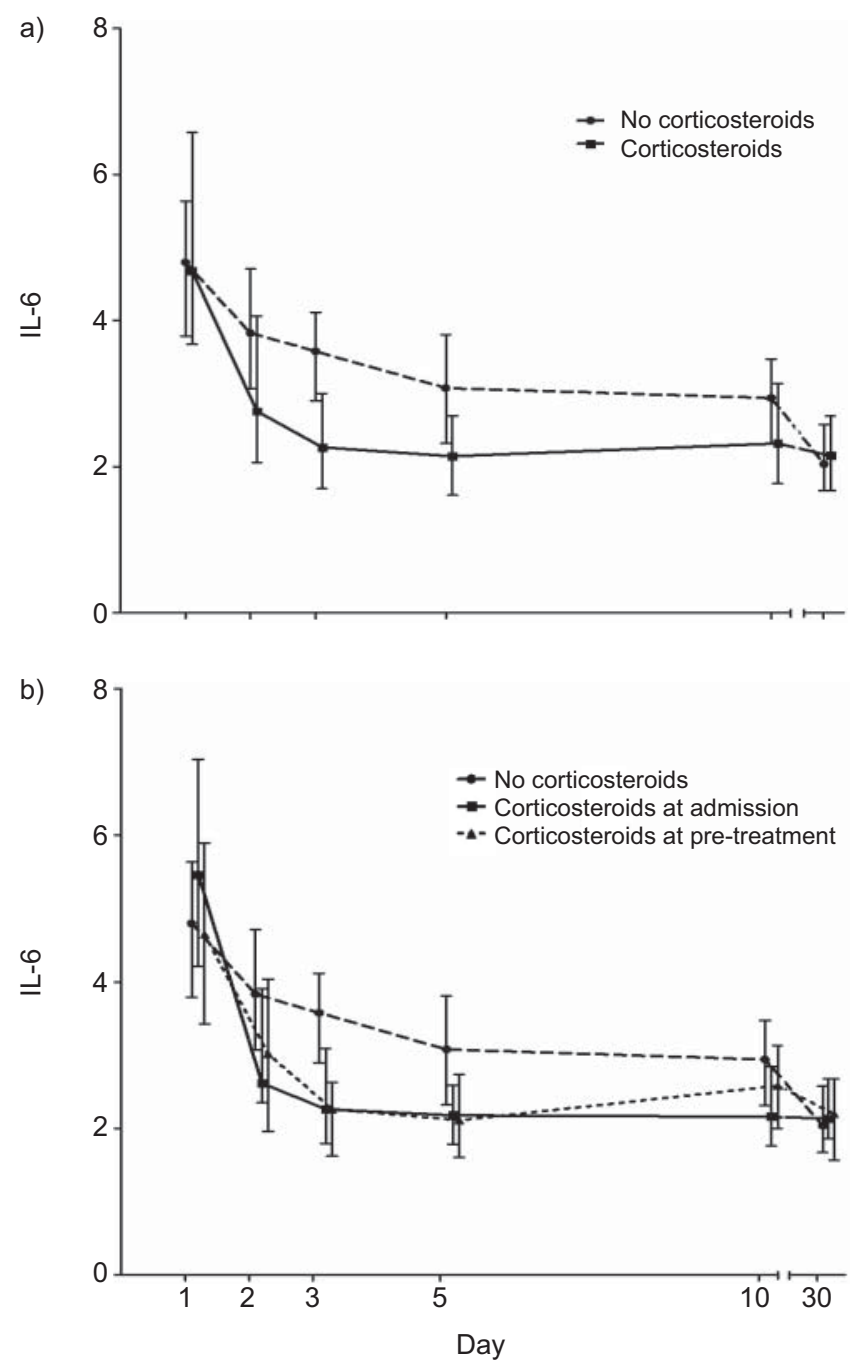

FIGURE 3. The effect of corticosteroids on interleukin (IL)-6 response. a) Median IL-6 levels (In scale) for patients on corticosteroid therapy that was started because of a pulmonary infection within 5 days prior to admission or at the emergency department during admission (corticosteroids) and patients who did not use corticosteroids at all (no corticosteroids) are plotted. After adjusting for age, sex, Pneumonia Severity Index score, chronic obstructive pulmonary disease, IL-6 level on day 1 and pneumococcal pneumonia, corticosteroid therapy independently influenced IL-6 levels on day 3 ( $p<0.01$ ). b) Median IL-6 levels (In scale) for patients on corticosteroid therapy that was started because of the pulmonary infection $\leqslant 5$ days before hospital admission (corticosteroid pre-treatment), patients who started corticosteroids at the time of hospital admission (corticosteroids at admission) and patients who did not use corticosteroids at all (no corticosteroids) are plotted. No statistically significant differences in IL-6 levels on admission or during hospital stay were seen between the two groups on corticosteroid therapy. In both groups IL- 6 levels on day 3 were independently influenced by corticosteroids $(p<0.01)$.

In experimental in vitro response to S. pneumoniae, T-helper cell (Th) type 1 cytokine production (IL-12 and IFN- $\gamma$ ) was observed in the early phases of the disease [38-41]. However, this was not observed in patients, suggesting that Th1 cytokines do not participate in the acute phase response in human (pneumococcal) pneumonia. Alternatively, the putative Th1 response could occur in the very early phase of disease and levels of these cytokines returned to normal by the time of presentation to the ED.

The lack of finding a difference in distribution of cytokine polymorphisms between patients and controls could be that other patient characteristics are far more important in susceptibility and clinical course of CAP, such as age, medical history and causative microorganism. For the latter, it is known that CAP caused by a specific microorganism is associated with cytokine polymorphism [26, 42, 43]. Although we were able to identify causative microorganisms in $64 \%$ of the patients, the groups of patients with a single causative microorganism were too small for further analysis.

In healthy volunteers, polymorphisms in cytokine encoding genes determine the magnitude of the endotoxin induced systemic cytokine response [44-46]. We found no effect of genetic polymorphisms in cytokine genes on the ultimate levels or kinetics of the cytokine response in CAP, probably due to an overwhelming inflammatory response caused by a high amount of microorganisms, endotoxins and other pro-inflammatory proteins. In healthy volunteers, the cytokine response is stimulated by small doses of endotoxins and without living microorganisms or other pro-inflammatory proteins. This might explain the polymorphism-dependent differences in cytokine responses observed in healthy volunteers but not in patients.

Post hoc analysis revealed a significant effect of the start of corticosteroid therapy on the levels of IL-6, IL-1RA, and MCP (corrected for possible confounders). This decrease is most probably due to the anti-inflammatory properties of the corticosteroids and is in agreement with previous literature $[47,48]$. Although this may also be due to the effect of upregulated regulatory cytokines. IL-10 levels were higher at admission in patients on pre-hospital corticosteroid therapy compared with patients who weren't. The majority of this group of patients (74\%) suffered from COPD, which is regarded as a chronic inflammatory disease. It is possible that during the early phase of $\mathrm{CAP}$, regulatory cytokines are already in an activated state in patients with COPD on corticosteroid therapy $[49,50]$. Patients treated with corticosteroids shortly before admission showed the same decrease in IL-6 levels compared with patients who started corticosteroids at admission. This suggests that a low dose of corticosteroids may not be sufficient to decrease cytokine levels. Prospective larger scale trials are warranted to evaluate the effect of corticosteroids on cytokine kinetics and outcome.

In pneumococcal pneumonia, IL-6 and IL-1RA play a more prominent role in the innate immune response than in CAP caused by other microorganisms. One reason could be that S. pneumoniae is an extracellular pathogen and causes a more invasive disease [51]. A number of the other causative pathogens are intracellular bacteria and therefore the nature of the cytokine response will differ $[52,53]$. An alternative, but not mutually exclusive explanation is that the clinical course of pneumococcal pneumonia is more rapid and severe; patients would therefore present themselves at the hospital shortly after or at the peak of the cytokine storm. However, we did not find differences in the length of the period between the first clinical signs until hospital admission in pneumococcal and nonpneumococcal patients. 
Inadequate antibiotic treatment may influence the kinetics of the cytokine response. The causative pathogen was identified in $64 \%$ of the patients and in the majority of the patients adequate treatment was given. Inadequate treatment was initially only given to patients with atypical bacteria, such as M. pneumoniae. These patients normally have a mild disease probably with a modest cytokine storm and therefore the results will be mildly influenced.

The main limitations of this study are the different nature of the causative microorganisms, the lack of a validated clinical score to reflect severity of disease during hospital stay and a lack of power to predict clinical outcome. As shown, the cytokine response differs in patients dependent on the causative microorganism of CAP. Relationships between cytokine levels, polymorphisms and clinical characteristics could be clearer in a group of patients with one causative microorganism. Our study is underpowered to perform analysis in subgroups (for example, patients with pneumococcal pneumonia). Another limitation is the lack of a validated score system for severity of disease as we were interested in the level of cytokine response in relation to severity of disease. PSI at admission is a score to predict mortality and appeared not to be useful as a score for severity of disease during hospital stay. Finally, due to a low mortality rate and few ICU admissions we were unable to show an association between cytokine levels and outcome.

In conclusion, we show that IL-6 and IL-8, together with IL-1RA and IL-10 act as acute phase proteins in CAP; IL- 6 is a more accurate biomarker for the follow-up of CAP than CRP. Genotype does not influence the levels of cytokine production in CAP. Furthermore, the systemic cytokine response is influenced by the causative microorganism and the cytokine response can be reduced by the use of corticosteroids in patients with CAP.

\section{STATEMENT OF INTEREST}

None declared.

\section{ACKNOWLEDGEMENTS}

We would like to thank R. van Hemert (Dept of Clinical Chemistry, St. Antonius Hospital, Nieuwegein, the Netherlands) and B. de Jong (Dept of Medical Microbiology and Immunology, St. Antonius Hospital, Nieuwegein, the Netherlands) for their expert technical assistance.

\section{REFERENCES}

1 Garau J, Baquero F, Perez-Trallero E, et al. Factors impacting on length of stay and mortality of community-acquired pneumonia. Clin Microbiol Infect 2008; 14: 322-329.

2 Kolling UK, Hansen F, Braun J, et al. Leucocyte response and antiinflammatory cytokines in community acquired pneumonia. Thorax 2001; 56: 121-125.

3 Tuomanen E, Rich R, Zak O. Induction of pulmonary inflammation by components of the pneumococcal cell surface. Am Rev Respir Dis 1987; 135: 869-874.

4 van der Poll T, de Waal MR, Coyle SM, et al. Antiinflammatory cytokine responses during clinical sepsis and experimental endotoxemia: sequential measurements of plasma soluble interleukin (IL)-1 receptor type II, IL-10, and IL-13. J Infect Dis 1997; 175: 118-122.

5 Ziegler-Heitbrock HW, Passlick B, Kafferlein E, et al. Protection against lethal pneumococcal septicemia in pigs is associated with decreased levels of interleukin-6 in blood. Infect Immun 1992; 60: 1692-1694.

6 Takashima K, Tateda K, Matsumoto T, et al. Role of tumor necrosis factor $\alpha$ in pathogenesis of pneumococcal pneumonia in mice. Infect Immun 1997; 65: 257-260.

7 Rijneveld AW, Florquin S, Branger J, et al. TNF- $\alpha$ compensates for the impaired host defense of IL-1 type I receptor-deficient mice during pneumococcal pneumonia. J Immunol 2001; 167: 5240-5246.

8 Kerr AR, Irvine JJ, Search JJ, et al. Role of inflammatory mediators in resistance and susceptibility to pneumococcal infection. Infect Immun 2002; 70: 1547-1557.

9 Preston JA, Beagley KW, Gibson PG, et al. Genetic background affects susceptibility in nonfatal pneumococcal bronchopneumonia. Eur Respir J 2004; 23: 224-231.

10 Khan $A Q$, Shen $Y, W u Z Q$, et al. Endogenous pro- and antiinflammatory cytokines differentially regulate an in vivo humoral response to Streptococcus pneumoniae. Infect Immun 2002; 70: 749761.

11 Zeng X, Moore TA, Newstead MW, et al. Intrapulmonary expression of macrophage inflammatory protein $1 \alpha$ (CCL3) induces neutrophil and NK cell accumulation and stimulates innate immunity in murine bacterial pneumonia. Infect Immun 2003; 71: 1306-1315.

12 Amano H, Morimoto K, Senba M, et al. Essential contribution of monocyte chemoattractant protein-1/C-C chemokine ligand-2 to resolution and repair processes in acute bacterial pneumonia. J Immunol 2004; 172: 398-409.

13 Puren AJ, Feldman C, Savage N, et al. Patterns of cytokine expression in community-acquired pneumonia. Chest 1995; 107: 1342-1349.

14 Majetschak M, Flohe S, Obertacke U, et al. Relation of a TNF gene polymorphism to severe sepsis in trauma patients. Ann Surg 1999; 230: 207-214.

15 Dehoux MS, Boutten A, Ostinelli J, et al. Compartmentalized cytokine production within the human lung in unilateral pneumonia. Am J Respir Crit Care Med 1994; 150: 710-716.

16 Tateda K, Moore TA, Deng JC, et al. Early recruitment of neutrophils determines subsequent $\mathrm{T} 1 / \mathrm{T} 2$ host responses in a murine model of Legionella pneumophila pneumonia. I Immunol 2001; 166: 3355-3361.

17 Kolsuz M, Erginel S, Alatas O, et al. Acute phase reactants and cytokine levels in unilateral community-acquired pneumonia. Respiration 2003; 70: 615-622.

18 Monton C, Torres A, El-Ebiary M, et al. Cytokine expression in severe pneumonia: a bronchoalveolar lavage study. Crit Care Med 1999; 27: 1745-1753.

19 Fernandez-Serrano S, Dorca J, Coromines M, et al. Molecular inflammatory responses measured in blood of patients with severe community-acquired pneumonia. Clin Diagn Lab Immunol 2003; 10: 813-820.

20 Glynn P, Coakley R, Kilgallen I, et al. Circulating interleukin 6 and interleukin 10 in community acquired pneumonia. Thorax 1999; 54: 51-55.

21 Antunes G, Evans SA, Lordan JL, et al. Systemic cytokine levels in community-acquired pneumonia and their association with disease severity. Eur Respir J 2002; 20: 990-995.

22 von Dossow V, Rotard K, Redlich U, et al. Circulating immune parameters predicting the progression from hospital-acquired pneumonia to septic shock in surgical patients. Crit Care 2005; 9: R662-R669.

23 Arnalich F, Lopez-Maderuelo D, Codoceo R, et al. Interleukin-1 receptor antagonist gene polymorphism and mortality in patients with severe sepsis. Clin Exp Immunol 2002; 127: 331-336.

24 Schluter B, Raufhake C, Erren M, et al. Effect of the interleukin-6 promoter polymorphism $(-174 \mathrm{G} / \mathrm{C})$ on the incidence and outcome of sepsis. Crit Care Med 2002; 30: 32-37. 
25 Gallagher PM, Lowe G, Fitzgerald T, et al. Association of IL-10 polymorphism with severity of illness in community acquired pneumonia. Thorax 2003; 58: 154-156.

26 Schaaf BM, Boehmke F, Esnaashari H, et al. Pneumococcal septic shock is associated with the interleukin-10-1082 gene promoter polymorphism. Am J Respir Crit Care Med 2003; 168: 476-480.

27 Sutherland AM, Walley KR, Manocha S, et al. The association of interleukin 6 haplotype clades with mortality in critically ill adults. Arch Intern Med 2005; 165: 75-82.

28 Schaaf B, Rupp J, Muller-Steinhardt M, et al. The interleukin-6 -174 promoter polymorphism is associated with extrapulmonary bacterial dissemination in Streptococcus pneumoniae infection. Cytokine 2005; 31: 324-328.

29 Stuber F, Petersen M, Bokelmann F, et al. A genomic polymorphism within the tumor necrosis factor locus influences plasma tumor necrosis factor- $\alpha$ concentrations and outcome of patients with severe sepsis. Crit Care Med 1996; 24: 381-384.

30 Fine MJ, Singer DE, Hanusa $\mathrm{BH}$, et al. Validation of a pneumonia prognostic index using the MedisGroups Comparative Hospital Database. Am J Med 1993; 94: 153-159.

31 Fine MJ, Auble TE, Yealy DM, et al. A prediction rule to identify low-risk patients with community-acquired pneumonia. $N$ Engl J Med 1997; 336: 243-250.

32 Endeman $\mathrm{H}$, Schelfhout V, Voorn GP, et al. Clinical features predicting failure of pathogen identification in patients with community acquired pneumonia. Scand J Infect Dis 2008; 40: 1-6.

33 Gabay C, Kushner I. Acute-phase proteins and other systemic responses to inflammation. $N$ Engl J Med 1999; 340: 448-454.

34 Steinhauser ML, Hogaboam CM, Kunkel SL, et al. IL-10 is a major mediator of sepsis-induced impairment in lung antibacterial host defense. J Immunol 1999; 162: 392-399.

35 Terashima T, Amakawa K, Matsumaru A, et al. BAL induces an increase in peripheral blood neutrophils and cytokine levels in healthy volunteers and patients with pneumonia. Chest 2001; 119: 1724-1729.

36 Bruunsgaard H, Skinhoj P, Qvist J, et al. Elderly humans show prolonged in vivo inflammatory activity during pneumococcal infections. J Infect Dis 1999; 180: 551-554.

37 van der Poll T, Keogh CV, Guirao X, et al. Interleukin-6 genedeficient mice show impaired defense against pneumococcal pneumonia. J Infect Dis 1997; 176: 439-444.

38 Arva E, Andersson B. Induction of phagocyte-stimulating and Th1-promoting cytokines by in vitro stimulation of human peripheral blood mononuclear cells with Streptococcus pneumoniae. Scand J Immunol 1999; 49: 417-423.

39 Arva E, Andersson B. Kinetics of cytokine release and expression of lymphocyte cell-surface activation markers after in vitro stimulation of human peripheral blood mononuclear cells with Streptococcus pneumoniae. Scand J Immunol 1999; 49: 237-243.
40 Lauw FN, Branger J, Florquin S, et al. IL-18 improves the early antimicrobial host response to pneumococcal pneumonia. J Immunol 2002; 168: 372-378.

41 Rubins JB, Pomeroy C. Role of $\gamma$ interferon in the pathogenesis of bacteremic pneumococcal pneumonia. Infect Immun 1997; 65: 2975-2977.

42 Schaaf B, Rupp J, Muller-Steinhardt M, et al. The interleukin-6 -174 promoter polymorphism is associated with extrapulmonary bacterial dissemination in Streptococcus pneumoniae infection. Cytokine 2005; 31: 324-328.

43 Temple SE, Lim E, Cheong KY, et al. Alleles carried at positions -819 and -592 of the IL10 promoter affect transcription following stimulation of peripheral blood cells with Streptococcus pneumoniae. Immunogenetics 2003; 55: 629-632.

44 Fijen JW, Tulleken JE, Hepkema BG, et al. The influence of tumor necrosis factor- $\alpha$ and interleukin-10 gene promoter polymorphism on the inflammatory response in experimental human endotoxemia. Clin Infect Dis 2001; 33: 1601-1603.

45 Fishman D, Faulds G, Jeffery R, et al. The effect of novel polymorphisms in the interleukin-6 (IL-6) gene on IL-6 transcription and plasma IL-6 levels, and an association with systemiconset juvenile chronic arthritis. J Clin Invest 1998; 102: 1369-1376.

46 Rivera-Chavez FA, Peters-Hybki DL, Barber RC, et al. Interleukin-6 promoter haplotypes and interleukin-6 cytokine responses. Shock 2003; 20: 218-223.

47 Keh D, Boehnke T, Weber-Cartens S, et al. Immunologic and hemodynamic effects of "low-dose" hydrocortisone in septic shock: a double-blind, randomized, placebo-controlled, crossover study. Am J Respir Crit Care Med 2003; 167: 512-520.

48 Monton C, Ewig S, Torres A, et al. Role of glucocorticoids on inflammatory response in nonimmunosuppressed patients with pneumonia: a pilot study. Eur Respir J 1999; 14: 218-220.

49 Vernooy JH, Kucukaycan M, Jacobs JA, et al. Local and systemic inflammation in patients with chronic obstructive pulmonary disease: soluble tumor necrosis factor receptors are increased in sputum. Am J Respir Crit Care Med 2002; 166: 1218-1224.

50 Pinto-Plata VM, Livnat G, Girish M, et al. Systemic cytokines, clinical and physiological changes in patients hospitalized for exacerbation of COPD. Chest 2007; 131: 37-43.

51 Mufson MA. Pneumococcal pneumonia. Curr Infect Dis Rep 1999; 1: $57-64$

52 Friedman H, Yamamoto Y, Newton C, et al. Immunologic response and pathophysiology of Legionella infection. Semin Respir Infect 1998; 13: 100-108.

53 Tanaka H, Narita M, Teramoto S, et al. Role of interleukin-18 and T-helper type 1 cytokines in the development of Mycoplasma pneumoniae pneumonia in adults. Chest 2002; 121: 1493-1497. 